\title{
LPE $\mathrm{Ga}_{a_{x}} \mathrm{In}_{1-x} \mathrm{~A} \mathrm{~s}_{1-y} \mathrm{Sb}_{y} / \operatorname{InP}$ 和 $\mathrm{G}_{\mathrm{a}_{x}} \mathrm{In}_{1_{-x}} \mathrm{~A} \mathrm{~s}_{1_{-y}} \mathrm{Sb}_{y} /$ InAs 的生长及其估价*
}

\author{
䴙秀英 高风升 王占国 韩文蓄 \\ (中国科学院半导体研究所，北京）
}

摘 要

将电负性差法估算的 $\mathrm{Ga}_{x} \mathrm{In}_{1-x} \mathrm{As}_{1-y} \mathrm{Sb}_{y} / \mathrm{InP}$ 和 $\mathrm{Ga}_{x} \mathrm{In}_{1-x} \mathrm{As}_{s_{-y}} \mathrm{Sb}_{y} / \mathrm{InAs}$ 的固相 和液相组份数据应用于液相外延生长,均获得了晶格匹配的村料, 并首次获得了波长 $\lambda>3.5 \mu \mathrm{m}$ 的 $\mathrm{Ga}_{x} \mathrm{In}_{1-x} \mathrm{As}_{1-y} \mathrm{Sb}_{y} / \mathrm{InAs}$ 异质外延材料. $\mathrm{Ga}_{x} \mathrm{In}_{1-x} \mathrm{As}_{\mathrm{1}_{1-y}} \mathrm{Sb} / \mathrm{InP}$ 的固 体组份为 $0.38<x<0.51,0.01<y<0.065$. 用 $\mathrm{X}$ 射线双晶行射、光学显微镜和 透射电镜 (TEM)、电学测试及低温光致发光实验对材料进行了估价. 结果歨明, 这 两种材料对于工作在 $1.3-1.6 \mu \mathrm{m}$ 和 $2.0-4.4 \mu \mathrm{m}$ 范围内的光电子器件及其集成有着 极其重要的应用前景。

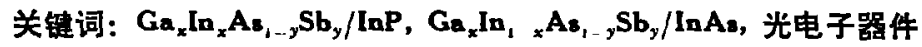

$\mathrm{Ga}_{x} \mathrm{In}_{1-x} \mathrm{As}_{\mathrm{1}_{-},} \mathrm{Sb}$, 是一种新的 III-V 族四元固溶体材料，它可以在不同的组份范围内分 别与 InP, GaSb 和 InAs 衬底的晶格相匹配, 波长范围覆盖 $1.3-4.4 \mu \mathrm{m}$. 在这一范围内, 光 纤的损失很小, 如果采用 $\mathrm{Ga}_{x} \mathrm{In}_{1_{-x}} A \mathrm{~s}_{\mathrm{I}_{-}} \mathrm{Sb}_{y}$ 一偂化物光纤系统 $(\lambda>2.0 \mu \mathrm{m})$, 有可能实现全球 无中继的光通讯 ${ }^{[1]}$.

另一方面,由于 GaAs-InSb 体系的固溶体材料与 III-V 族中其他的体系相比，有着较小 的电子有效质量 $m_{\mathrm{e}}^{*}$, 因而电子迁移率很高, 非常适合于制作 FET, HEMT 等超高速电子器 件.

尽管 $\mathrm{Ga}_{x} \mathrm{In}_{\mathrm{1}_{-}} A \mathrm{As}_{\mathrm{t}_{-}}, \mathrm{Sb}$, 材料具有很大的潜在的重要性及应用前景, 但多年来关于其生长 和性质研究的报道一直不多。只是近年来，随着重金属氮化物光纤研制工作的突破以及在解 决这一体系的所谓“混溶隙”问题方面取得了进展, 关于这一材料的生长及其器件的研制工作 才越来越受到人们的重视.

以前的报道中，人们主要集中在 $\mathrm{GaSb}$ 祄底上的生长 ${ }^{[2-a]}$. 关于 $\mathrm{Ga}_{x} \mathrm{In}_{1-x} \mathrm{As}_{\mathrm{1}_{-},} \mathrm{Sb}, / \mathrm{InAs}$

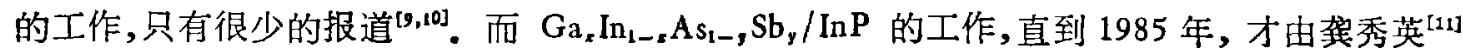

本文 1988 年 6 月 13 日收到, 1989 年3 月4 日收到修改穗.

*参加过本工作的还有卢文宏、赵海洋、李成基、王万年、崔玉德和王玉田同志作了固体组份分析、电学测试、伐歇能谱 分析及 $\mathrm{X}$ 射线双晶衍射测量;褚一鸣、陆珢华、王风莲同志为我们作了透射电子显微镜分析; 杨保华、刘学锋同志作了 有益的协助, 在此作者一并表示感谢. 
等首先报道的.

不少学者 ${ }^{[3,12,13]}$ 认为,在 $\mathrm{Ga}_{x} \mathrm{In}_{1-x} A s_{1-y} 9 \mathrm{~b}_{y}$ 体系中存在着一个严重的问题，即所谓 “混溶 隙”问题. 事实上, 长期以来, LPE $\mathrm{Ga}_{x} \mathrm{In}_{1-x} \mathrm{As}_{1_{-y}} \mathrm{Sb}_{y} / \mathrm{GaSb}$ 中 In 的原子分数不能突破 0.18 , 直到 1985 年, Dewinter ${ }^{[0]}$ 等人才扩展到 0.22 , 刚刚进人了“混溶隙”的范围. 但迄今为止, $\mathrm{Ga}_{x} \mathrm{ln}_{1-x} \mathrm{As}_{\mathrm{l}_{-}, \mathrm{Sb}} / \mathrm{AlGaAsSb} / \mathrm{GaSb}$ 激光翟的波长还未能超过 $2.4 \mu \mathrm{m}$, 仍然受到“混溶隙”的阻 拦。供秀英等人关于 $\mathrm{Ga}_{x} \mathrm{In}_{1-x} \mathrm{As}_{\mathrm{t}_{-y}} \mathrm{Sb}_{y} / \mathrm{InP}$ 的工作使这一材料的短波限从 $1.7 \mu \mathrm{m}$ 伸展到 $1.3 \mu \mathrm{m}$.

本文将较详细的报道在我们实验室生长的 $\mathrm{LPE} \mathrm{Ga}_{x} \mathrm{In}_{1-x} \mathrm{As} \mathrm{s}_{1-y} \mathrm{Sb}$, / InP 的结构、电学和

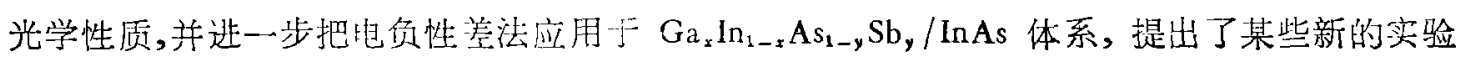
结果及对打破所谓“鼬溶谅”问题的设態.

\section{一、LPE 生长实验}

$\mathrm{Ga}_{x} \mathrm{In}_{1_{-x}} \mathrm{As}_{1_{-}} \mathrm{Sb}$ 外延材料是在管规的水平滑动系统中生长的,采用玻璃态石墨舟. Pd 扩 散的 $\mathrm{H}_{2}$ 用作保护气氛. 你材料题 $7 \mathrm{NIn}, 6 \mathrm{NSb}$ 以及非掺杂的 GaAs 和 InAs. 祄底为掺 $\mathrm{Fe}$ 半绝缘 $\mathrm{InP}$ 和非掺杂 $\operatorname{InAs}\left(n \sim 10^{26} \mathrm{~cm}^{-3}\right)$ 单晶, 均为 $(100)$ 取向. 生长温度为 $650^{\circ} \mathrm{C}$, 降温 速率为 $0.5^{\circ} \mathrm{C} / \mathrm{min}$. 为了犾得晶格四配的外延层, 各组元分凝系数的选取必须合理, 称量必须 准确无误.

实际上,由于各组元的分㴰系数即使在相同的溶剂中也是随温度而变化的,因此，当采用 不同的生长温度付，应选用不闰们分凝系数数挹。

为了得到晶格匹配的外延层，生长溶液的均匀性也是非常重要的. 外延生长以前较长时 间的热处理不仅促你组元的分了任溶液中分布均匀, 而且即使出现 $\mathrm{Ga}-\mathrm{Sb}, \mathrm{In}-\mathrm{Sb}$ 篮分子的 缔合,也不会影响晶格匹配的外延层的生长.

\section{二、LPE $\mathrm{Ga}_{x} \ln _{1-x} \mathrm{As}_{1-y} \mathrm{Sb}_{y} / \mathrm{InP}$ 和 $\mathrm{Ga}_{x} \mathrm{In}_{1-x} \mathrm{As}_{1-y} \mathrm{Sb}_{y} / \mathrm{InAs}$ 的组份}

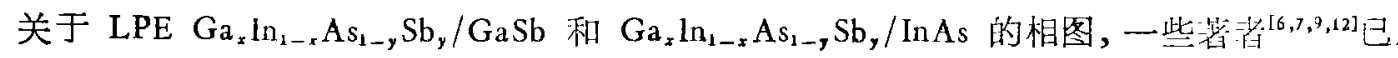
经研究过了. 他们采用的理论计算法大都是规则溶液近似法, 在液相外延生长测定中, 用的是 失重法或直接观察法。

我们用的是电负性差法. 曾先利用二元化合物的晶格常数与元素电负性差的关系 ${ }^{[14}$ 估算

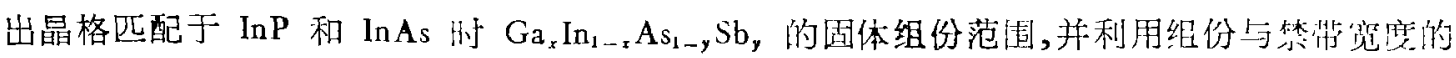
关系 ${ }^{[151}$ 确定预定带宽(视所需的波长而定) 时相应的固体组份, 然后根据各组元在生长温度下

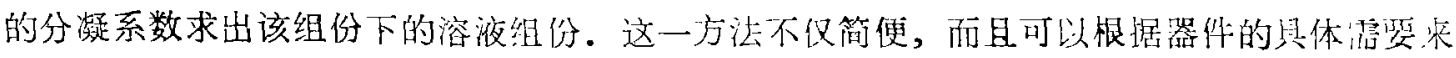
设计固溶体的组分, 并直接用称重法犾得晶格匹配的材料. 我们的液相外延生长实验证实, 这 一方法是可行的.

图 1(a) 和 (b) 分别示出了生长 $\mathrm{Ga}_{a_{x}} \mathrm{In}_{1_{-}} \mathrm{As}_{1_{-}}, \mathrm{Sb}_{y} / \mathrm{InP}$ 时溶液中 $\mathrm{As}$ 和 $\mathrm{G}_{2}$ 的原子分数与

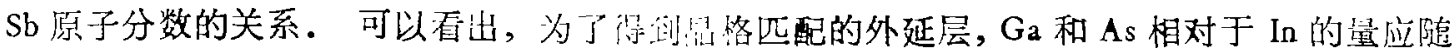
$\mathrm{Sb}$ 量的增加而增扣. 也就是说, $\mathrm{Ga}_{\mathrm{r}} \mathrm{In}_{1_{-x}} \mathrm{As}_{\mathrm{s}_{1-y}} \mathrm{Sb} / \mathrm{InP}$ 的组妢应从 $\ln \mathrm{GaAs}_{\mathrm{s}}$ 移向 $\mathrm{GaAsSb}$ ，与， 屶俋性差法算得的结果一致。

固体组份是用 JXA-3A 型电子探针 $\mathrm{X}$ 射线微分析仪测定的。分析时用的 $\mathrm{X}$ 射线为 31 - 


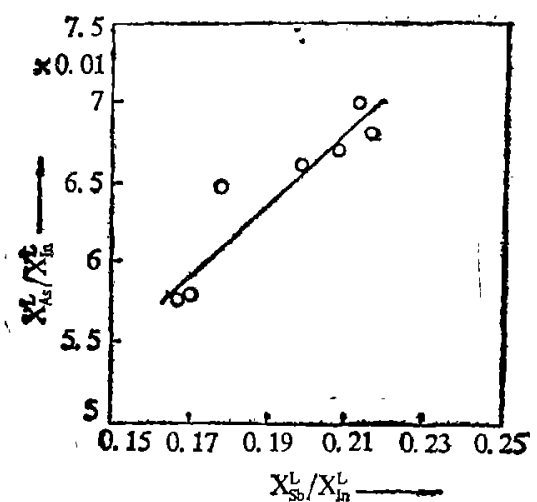

(a)

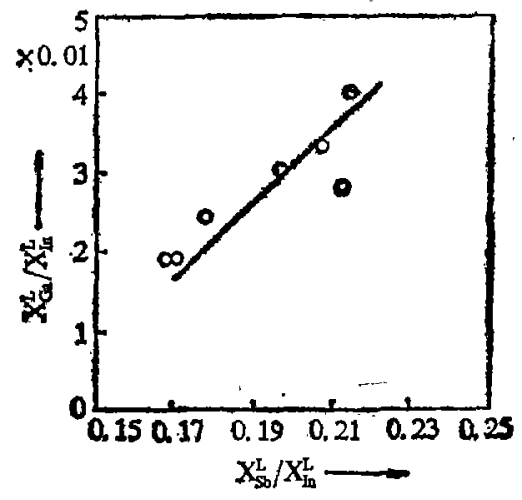

(b)

图 $1650^{\circ} \mathrm{C}$ 下， $\mathrm{LPE} \mathrm{Ga} \mathrm{In}_{\mathrm{A}_{-x}} \mathrm{As} \mathrm{s}_{1-y} \mathrm{Sb}_{y} / \mathrm{In} \mathrm{P}$ 溶液中 $\mathrm{As}$ 和 $\mathrm{Ga}$ 的原子分数与 $\mathrm{Sb}$ 原子分数的关系

表 1 LPE Ga $\mathrm{In}_{\mathbf{x}} \mathrm{n}_{\mathrm{x}} \mathrm{As} \mathrm{s}_{1-y} \mathrm{Sb} \mathrm{b}_{\mathrm{y}} / \mathrm{In} \mathrm{As}$ 的组份和波长

\begin{tabular}{l|c|c|c|c|c|c}
\hline \multirow{2}{*}{ 样品号 } & \multicolumn{2}{|c|}{$x$} & \multicolumn{2}{|c|}{$y$} & \multicolumn{2}{c}{$\lambda(\mu \mathrm{m})$} \\
\cline { 2 - 7 } & 电负性差法 & 实 验 & 电负性差法 & 实 验 & 电负性差法 & 内插法 \\
\hline As-402 & 0.11 & 0.12 & 0.15 & 0.15 & 3.78 & 3.78 \\
As-404 & 0.10 & 0.09 & 0.08 & 0.08 & 3.47 & 3.45 \\
As-502 & 0.09 & 0.08 & 0.13 & 0.13 & 3.95 & 3.92 \\
As-505 & 0.08 & 0.08 & 0.12 & 0.10 & 3.70 & 3.70 \\
As-603 & 0.10 & 0.11 & 0.10 & 0.10 & 3.37 & 3.38 \\
As-607 & 0.09 & 0.09 & 0.12 & 0.11 & 3.58 & 3.59 \\
As-609 & 0.09 & 0.10 & 0.10 & 0.10 & 3.43 & 3.17 \\
\hline
\end{tabular}

$\mathrm{Ga}_{2}: \mathrm{Ka} ， 33-\mathrm{As}: \mathrm{Ka} ， 49-\mathrm{In}: \mathrm{La}$ 和 51-Sb:La. 加速电压为 $25 \mathrm{kV}$ ，外延层厚 6-10 $\mu \mathrm{m}$. 进行 了吸收、荧光和原子序数等项校正。结果表明, $\mathrm{LPE} \mathrm{Ga}_{x} \mathrm{In}_{1-x} \mathrm{As}_{1_{-y}} \mathrm{Sb}_{y} / \mathrm{InP}$ 的组份范围为 $0.38<x<0.51,0.01<y<0.065$, 与作者在西德马普固体所时得到的数据基本一致.

表 1 列出了 $\mathrm{Ga}_{x} \mathrm{In}_{1_{-}} \mathrm{As}_{1_{-}}, \mathrm{Sb}, \mathrm{InAs}$ 的组份和波长的数据. 其中组份值是用电负性差法 预先确定的, 实验值是从相应的溶洨中生长出外延片,再由电子微探针测定的; 而列出的波长 数据是用电负性差法和内插法 ${ }^{[16]}$ 分别推算得到的.

可以看出, 用电负性差法预先确定的组份与实验测定值相当符合，两种方法求出的波长数 据也符合得很好. 从而证明，电负性差法对于 $\mathrm{Ga}_{x} \mathrm{In}_{1_{-x}} \mathrm{As}_{1_{-},} \mathrm{Sb}_{y} / \mathrm{In} \mathrm{P}$ 和 $\mathrm{Ga}_{2_{x}} \mathrm{In}_{1_{-x}} \mathrm{As}_{1_{1-y}} \mathrm{Sb}_{y} /$ InAs 体系都是适用的. 结果也说昒, 以 InAs 作为衬底来生长波长超过 $3.5 \mu \mathrm{m}$ 的 $\mathrm{Ga}_{\mathbf{x}} \mathrm{In}_{1-\text { ， }}$ ， $\mathrm{As}_{\mathrm{1}_{-}, \mathrm{Sb}} \mathrm{Sb}$ 材料是可以实现的. 这无论对于低损耗远距离光纤通信还是作为其他应用的中红外 探测器的研制, 都具有极其重要的意义.

如上所述,由于所谓“混溶隙”的限制, 至今未能在 GaSb 衬底上获得波长超过 $2.5 \mu \mathrm{m}$ 的 $\mathrm{Ga}_{x} \mathrm{In}_{1-x} A s_{1-}, \mathrm{Sb}_{y}$ 材料. 然而, ${ }_{\mathrm{GaSb}}$ 和 InAs 的晶格常数是十分接近的, 从晶格匹配的角度 看,在 $\mathrm{GaSb}$ 衬底上应该能生长出富 InAs 的材料, 反之亦然. 至今未能成功, 至少其原因之 
一是液相外延时溶液组份选择得不合理。因为在固溶体材料的生长中，与衬底晶格严央失配 必将影响新晶核的形成自由能. 反之,如果这一条件可以满足,则在此组份范围内混溶隙可能 并不存在或范围很小. 如果采用电负性差法预先设计出与衬底晶格匹配时的固相和液相的组 份，直接用称重法精确称䥣各组元的量，并掌握好外延生长过程，则某些学渚预言的关于 $\mathrm{Ga}_{x} \mathrm{In}_{1-x} \mathrm{As}_{1_{-y}} \mathrm{Sb}_{y}$ 体系的所谓“混溶隍”的范围有可能被大大缩小。

\section{三、表面形貌和结构完整性}

外延层的表面形貌和结构它整性是表征材料质量的一个重要方面. 我们是采用光学金相 显微镜和透射电子显微镜 (TEM) 研究的. 结果发现, 在暃格匹配的组份范围内生长的外延 层, 表面呈镜面光滑, 异质结界面也是平坦的, 没有出现失配位错. 这表明不仅外延层和衬底 间的热膨胀系数相差很小，而且两者间的晶格失配度也是很小的. $\mathrm{Ga}_{x} \mathrm{In}_{1_{-x}} A s_{1_{-y}} \mathrm{Sb}_{y} / \mathrm{InP}$ 的

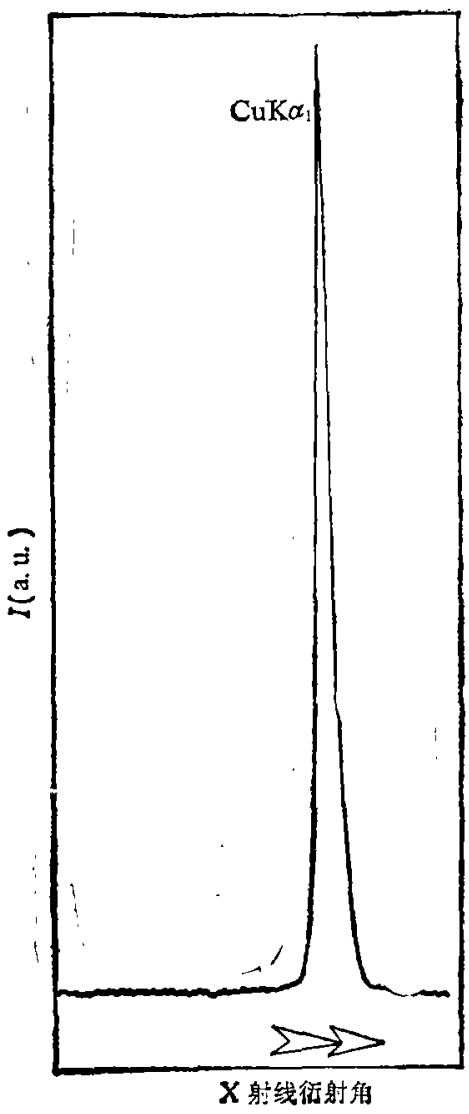

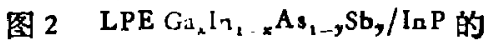
典型的 $\mathrm{X}$ 射线双晶行射回摆曲线

(晶格失配度 $\sim 2 \times 10^{-4}$ )

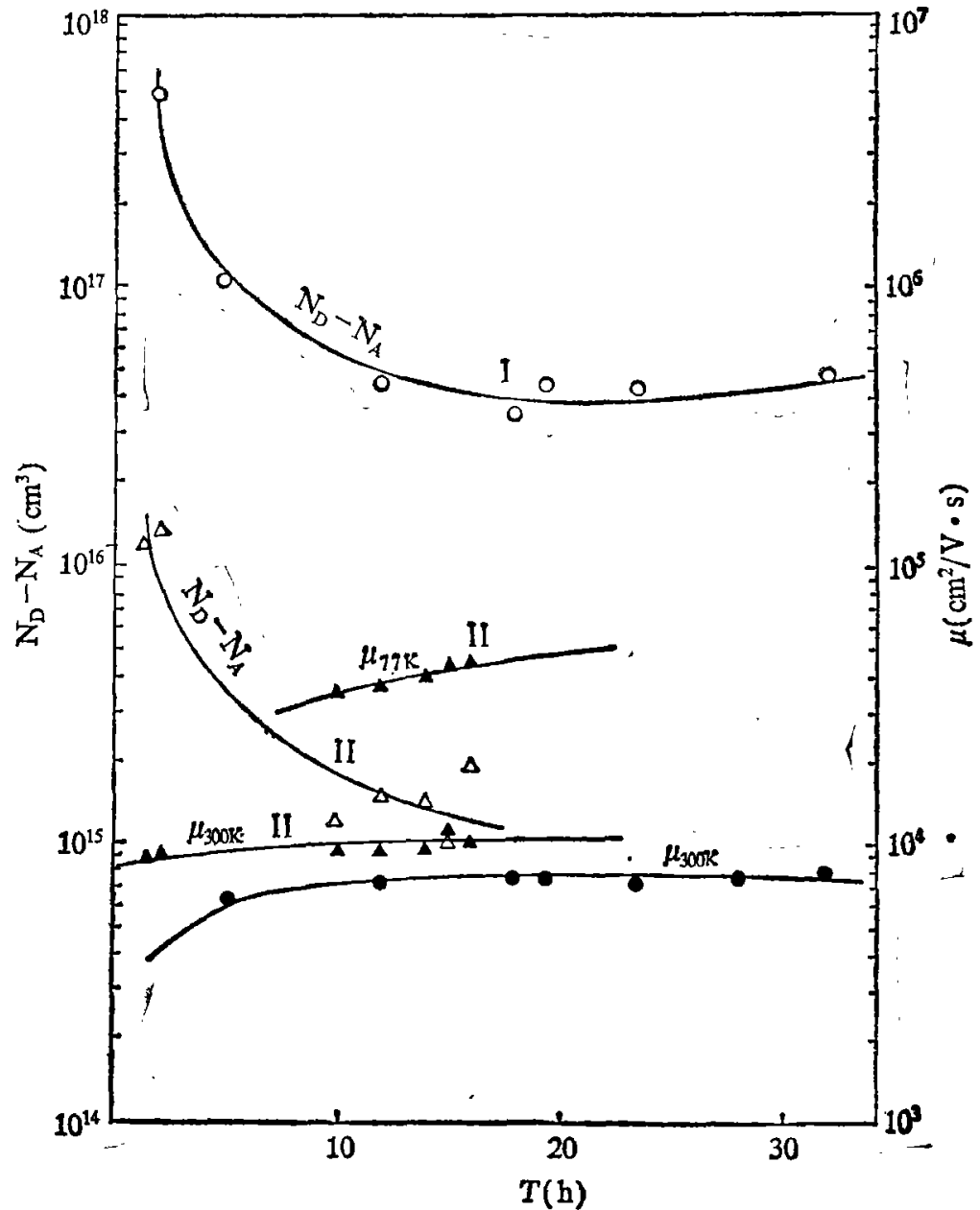

图 $3 \mathrm{Ga}_{x} \mathrm{In}_{1} \times \mathrm{As}_{1,}, \mathrm{Sb} / \mathrm{InP}$ 的电学参数与 溶液焙烧时间的关系 
晶格失配度是用 $X$ 射线双晶衍射法测定的. 第 1 晶体用 $\operatorname{Si}(422) ， \mathrm{CuK} \alpha_{1}$ 辐射。衍射仪的角 分辨率为 $5 \times 10^{-5}$. 图 2 是一个样品的 $\mathrm{X}$ 射线双晶衍射迴摆曲线,衍射矢量为 400 . 从图中看 出, 衬底和外延层的峰距离很小, 以致重叠在一起, 从两者重叠在一起的结果估计,其晶格失配 度为 $2 \times 10^{-4}$. 外延峰梢宽, 可能是由于外延层较厚 $(\sim 10 \mu \mathrm{m})$, 存在某些组份变化的缘做.

\section{四、电 学 性 质}

外延层的电学性质是用 Van der pauw 法研究的. 表 2 列出了 $\mathrm{Ga}_{x} \mathrm{In}_{1_{-x}} \mathrm{As}_{1_{-y}} \mathrm{Sb}_{y} / \mathrm{InP}$ 的 具型的电学数据. 图 3 示出了两组样品的载流子浓度和电子迁移率与总的溶液焙烧时间的关 系。这些样品是在不同的条件下生长的. 显然, 随着累计焙烧时间的增加，直到 $15 \mathrm{~h}$ ，两组样 品的载流子浓度都下降, 电子迁移率都上升. 说明焙烧溶液对提高外延层的纯度是有效的, 溶 液中的挥发性杂质和氧化物可以被除去; 但当总时间超过 $15 \mathrm{~h}$ 后,进一步的提纯效果就不明显 了, 可能是由于生长舟和氢气纯度的限制.

表 2 LPE Ga $\operatorname{In}_{1-x} A s_{1-y} S_{y} / I_{n} P$ 的电学性质

\begin{tabular}{|c|c|c|c|c|c|}
\hline \multirow{2}{*}{ 样品号 } & \multirow{2}{*}{$\begin{array}{c}\text { 孯 雍 } \\
(\mu \mathrm{m})\end{array}$} & \multicolumn{2}{|c|}{$300 \mathrm{~K}$} & \multicolumn{2}{|c|}{$77 \mathrm{~K}$} \\
\hline & & $\left(\begin{array}{c}n \\
\left(\mathrm{~cm}^{-3}\right)\end{array}\right.$ & 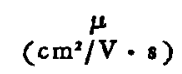 & $\left(\begin{array}{c}n \\
\left(\mathrm{~cm}^{-3}\right)\end{array}\right.$ & $\stackrel{\mu}{\left(c m^{2}-V \cdot s\right)}$ \\
\hline P-701 & 9.9 & $1.33 \times 10^{16}$ & 9134 & 1 & 1 \\
\hline P-702 & 9.7 & $1.20 \times 10^{10}$ & 9024 & 1 & 1 \\
\hline P-705 & 6.0 & $1.01 \times 10^{15}$ & 10900 & $9.95 \times 10^{14}$ & 42653 \\
\hline P-706 & 6.5 & $1.28 \times 10^{15}$ & 9500 & $1.15 \times 10^{10}$ & 37100 \\
\hline P-707 & 6.0 & $2.00 \times 10^{10}$ & 9900 & $1.80 \times 10^{13}$ & 40420 \\
\hline P-708 & 6.6 & $1.49 \times 10^{13}$ & 9740 & $1.31 \times 10^{15}$ & 42020 \\
\hline
\end{tabular}

第二组样品是在不同的条件下生长的。采用了稀土元素掺杂剂. 载流子浓度已由 $n \sim 10^{16} \mathrm{~cm}^{-3}$ 降低到 $n \sim 10^{15} \mathrm{~cm}^{-3}$, 室温电子迁移率高达 $10^{4} \mathrm{~cm}^{2} / \mathrm{V} \cdot \mathrm{s}$. 最好的结果为: $n=1.01 \times 10^{15} \mathrm{~cm}^{-3}, \mu_{300 \mathrm{R}}=10900 \mathrm{~cm}^{2} / \mathrm{V} \cdot \mathrm{s}$ ，根据分析，掺人的稀土元素取代了 In 的亚晶 格. 另一方面, 它对 $\mathrm{O}_{2}, \mathrm{~S}, \mathrm{Si}$ 和 $\mathrm{C}$ 等杂质都有很强的化学亲合作用. 桸土元素的存在, 降低 了溶液中的杂质含量, 从而也降低了外延层中的残余杂质含量, 特别是施主杂质. 这就使外延 层的电子迁移率显著提高.

由此可见，尽管 $\mathrm{Ga}_{x} \mathrm{In}_{1_{-x}} \mathrm{As}_{1_{-y}} \mathrm{Sb}_{y} / \mathrm{InP}$ 的波长范围与 $\operatorname{InGaAsP} / \mathrm{InP}$ 的相当 (1.3$1.6 \mu \mathrm{m}$ ), 但电子迁移率却高得多. 这是因为 $\mathrm{InSb}$ 的电子有效质量比 $\operatorname{In} P$ 的小得多所致. [因 此, 对于在此波长范围内的高速器件及光电子器件的集成来说, $\mathrm{Ga}_{x} \operatorname{In}_{1_{-x}} A s_{1-y}, \mathrm{Sb}, / \mathrm{InP}$ 是一个 很优越的材料。

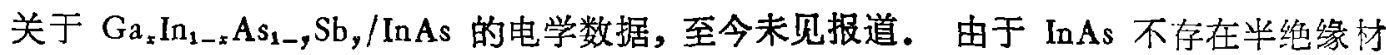
料,造成用常规的 Hall 法测试发生了困难,而红外反射光谱法适合于浓度较高的样品，而且 误差大达 $25 \%$. 积 . 为了解决这一问题,我们采用了选择腐蚀/Van der pauw 法, 即将 InAs 衬 底除去后再进行测试. 这一方法的关键是除尽祄底, 否则将影响数据的准确性. 对于未作桸 土掺杂的 LPE $\mathrm{Ga}_{x} \mathrm{In}_{1_{-}} A s_{1_{-}}, \mathrm{Sb}, / \mathrm{InAs}$ 材料(外延层厚 10 $\mu \mathrm{m}$ ), 典型的室温电学参数如下: 
$n \simeq\left(6 \times 10^{16}-2 \times 10^{17}\right) \mathrm{cm}^{-3}, \mu_{200 k} \simeq(14000-19000) \mathrm{cm}^{2} / \mathrm{V} \cdot \mathrm{s}$. 稀土元素掺人后的样品 为 $n=\left(5 \times 10^{15}-3 \times 10^{16}\right) \mathrm{cm}^{-3}, \mu_{300 K}=(20000-24800) \mathrm{cm} /{ }^{2} \mathrm{~V} \cdot \mathrm{s}$.

\section{五、低温光致发光实验}

LPE $\mathrm{Ga}_{x} \mathrm{In}_{1_{-}} \mathrm{As}_{1_{-y}} \mathrm{Sb}_{y} / \mathrm{InP}$ 的低温光致发光实验是用法国制造的 $\mathrm{H}_{25}$ 红外光柶单色仪 进行的, 实验用波长范围为 $1-2 \mu \mathrm{m}$, 狭琏宽度 $0.15 \mathrm{~mm}$ 时, 仪器分辨率优于 $20 \AA$.

表 $3 \mathrm{LPE} \mathrm{Ga} \mathrm{In}_{1-x} \mathrm{As}_{1-y} \mathrm{Sb}_{\boldsymbol{y}} / \mathrm{In} \mathrm{P}$ 的光致发光实验结果

\begin{tabular}{|c|c|c|c|c|c|c|}
\hline \multirow[b]{2}{*}{ 样品号 } & \multirow[t]{2}{*}{ PL 光谱 } & \multicolumn{3}{|c|}{ 半高宽 $(\mathrm{meV})$} & \multicolumn{2}{|c|}{ PL 强度比 } \\
\hline & & 带边发射带 A & 受主帯 B & 受主带C & $\mathrm{A} / \mathrm{B}$ & $A / C$ \\
\hline & P-701 & 10.80 & 39.1 & 1 & 25.4 & 1 \\
\hline & $P-705$ & 6.15 & 31.0 & 1 & 30.2 & 1 \\
\hline & $P-707$ & 7.30 & 30.8 & I & 4.83 & 1 \\
\hline & P-708 & 8.46 & 33.8 & 1 & 6.08 & 1 \\
\hline & P-709 & 14.50 & 33.8 & 54.2 & 14.29 & 21.4 \\
\hline & $P-29 *$ & 14.50 & 35.4 & 1 & 18.60 & 1 \\
\hline
\end{tabular}

- 西德马普固体所生长.

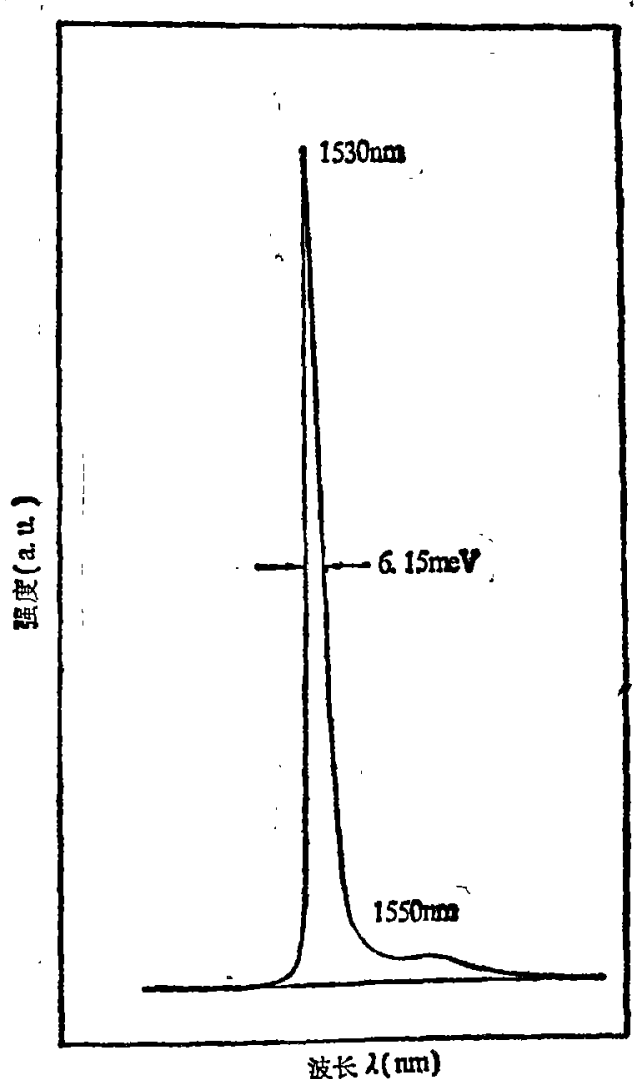

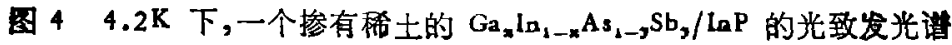


接收信号用的是液氮冷却的 $\mathrm{Ge}$ 探测器, 灵敏度为 $S \approx 4 \times 10^{8}-7 \times 10^{9} \mathrm{~V} / \mathrm{W}$. 激发光 源是美国光谱公司出产的 $\mathrm{Ar}^{+}$激光器. 测量时尽量降低激发强度, 以保证其测量精度和准确 性. 测试在 $4.2 \mathrm{~K}$ 下生行. 对于 6 个 $\mathrm{Ga}_{x} \mathrm{In}_{1-x} \mathrm{As}_{1_{-},} \mathrm{Sb}_{y} / \mathrm{InP}$ 样品, 固体组份范围为 $0.38<x<$ $0.51,0.01<y<0.065$, 结果如表 3 所示.

结果表明, 带边发射带 $\mathrm{A}$ 是由自由激子 (FE)、束缚在施主和受主上的激子以及施主-受 主 (D-A) 发光所组成. 未能分开的原因可能是各发光中心能量太接近或仪器分辩本领不够 高所致。

$\mathrm{B}, \mathrm{C}$ 发光带与自由到束缚 $(\mathrm{FB})$ 的浅受主发光相关. 根据分析, 受主主要是 $\mathrm{C}_{\mathbf{A s},}, \mathrm{Si}_{\mathbf{A s},}$, 而施主主要是 $O_{A_{s}}, S_{A_{s}}$ 和部分 $S i_{r_{s} s}$.

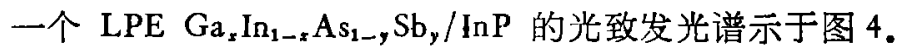

实验结果表明, 掺有稀土后的外延片, 纯度和完整性均有了明显提高, 这反映在 $\mathrm{A}$ 带的半 高宽降低到 $6.15 \mathrm{meV}$ 以及 $\mathrm{A} / \mathrm{B}$ 高达 30.2 (见图 4). 这一结果是目前关于 $\mathrm{Ga}_{x} \mathrm{In}_{1_{-x}} \mathrm{As}_{\mathrm{1}_{1-}}, \mathrm{Sb}$, 材料的最好结果(文献报道).

\section{六、结 论}

液相外延生长实验证实, 用电负性差法估算 $\mathrm{Ga}_{z} \mathrm{In}_{1_{-}} \mathrm{As_{1- } ,} \mathrm{Sb}_{y} / \mathrm{In} \mathrm{P}$ 和 $\mathrm{Ga}_{x} \mathrm{In}_{1_{-}} \mathrm{As}_{1_{-},}, \mathrm{Sb}_{y} /$ InAs 系统的固体和液体组份是可行的, 不仅方法简便, 而且可以根据光电子器件的实际需要 来进行设计.

实验证实, LPE $\mathrm{Ga}_{x} \mathrm{In}_{1-x} A s_{1-}, \mathrm{Sb}_{y} / \mathrm{InP}$ 的表面和结构完整性好, 晶格失配度小, 电子迁移 率高, 是波长在 $1.3-1.6 \mu \mathrm{m}$ 范围内的光电子器件及其集成的优越材料. 还采用 $(100)$ InAs 衬 底首次获得了波长超过 $3.5 \mu \mathrm{m}$ 的 $\mathrm{Ga}_{x} \mathrm{In}_{1-x} \mathrm{As}_{1_{-},} \mathrm{Sb}$, 材料, 经稀土掺杂后, 其电学参数达到: $n=\left(5 \times 10^{15}-3 \times 10^{16}\right) \mathrm{cm}^{-3}, \mu_{300 K}=(20000-24800) \mathrm{cm}^{2} / \mathrm{V} \cdot \mathrm{s}$ 的水平, 是第三代光通信 及中红外探测器的重要材料.

\section{参孝文 埱}

[1] France, R. W. et al., Electron. Lett., 20 (1984), 607.

[2] Kabayshi, N. et al., Jap. J. Appl. phys., 18 (1979), 2169.

[3] Kano. H. et al., Jap. J. Appl. Phys., 18 (1979), 2183.

[4] Dolginov, L. M. et al., Kristall und Technik, 13 (1978), 631.

[5] Tsang, W. T. et al., Appl. Phys. Latt., 46 (1985), 283.

[6] Dewinter, J. C. et 21., J. Electron Materiuls, 14 (1985), 729.

[7] Astles. M. et al., J. Appl. Phys., 60 (1986), 41.

[8] Wan der, Ziel, J. P. et al., J. Appl. Phys., 60 (1986), 4087.

[9] Sankaran, R. \& Antypas, G. A., J. Crystal Growth, 36 (1976), 198.

[10] Kobayashi, N. \& Horikoshi, Y., Jap. J. Appl. Phys., 20 (1981), 2253.

[11] Gong, X. Y. et al., 15th European Solid State Device Research Conference, Germany, $1985,202$.

[12] Nakijma, K. et al., J. Crystal Growth, 41 (1977), 87.

[13] String fellow, G. B. et al., J. Cryst. Growth, 58 (1982), 194.

[14] 咅秀英、高风开，稀有金属，7 (1983)， 21.

[15] 袋秀英、高风开, 金属(有色)，2 (1981)，11.

[16] Glisson, T. H. et al., J. Electron Mater, 7 (1978), 1.

[17] Bellov, A. G. et al., Sov. Phys. Solid Stare, 26 (1984), 84. 